\title{
Modified Integral Counting Method with Various Quenched Samples for Different Scintillators
}

\author{
Masato NAKAYAMA $^{1)}$, Masanori HARA ${ }^{1)}$, Masao MATSUYAMA ${ }^{1)}$ \\ and Kiyokazu HIROKAMI ${ }^{2}$ \\ 1) Hydrogen Isotope Research Center, Organization for Promotion of Research, University of Toyama \\ ${ }^{2)}$ Radioisotope Laboratory, Research and Development in Natural Sciences Center, \\ Administration Center for Promotion of Research, Organization for Promotion of Research, \\ University of Toyama \\ 3190 Gofuku, Toyama, Toyama Pref. 930-8555, Japan \\ Received Jun. 23, 2016; accepted Dec. 27, 2016
}

A modified integral counting method (MICM) with various quenched samples (MICM-VQ) has been investigated for its applicability for different scintillators using $\beta$ emitters, ${ }^{14} \mathrm{C}$ and ${ }^{35} \mathrm{~S}$. To assess the influence of scintillators, three sets of ${ }^{14} \mathrm{C}$ quenched standards and two ${ }^{35} \mathrm{~S}$ cocktail series were prepared. Two sets of ${ }^{14} \mathrm{C}$ quenched standards were used for the toluene-compatible scintillator, the other for the Ultima Gold ${ }^{\mathrm{TM}}$ scintillator. Sulfur-35 cocktail series were prepared with either Ecoscint ${ }^{\mathrm{TM}} \mathrm{XR}$ or Ultima Gold ${ }^{\mathrm{TM}} \mathrm{AB}$. The radioactivity of these samples was determined using the MICM-VQ, with the results conforming to assayed values. Hence the MICM-VQ can assay the radioactivity of sample cocktails with various scintillators and requires no standard sample.

Key Words: liquid scintillation counting, modified integral counting method, no quenched standard sets, different scintillators, point of convergence

[doi :10.12950/rsm.160623]

\section{Introduction}

Since the early 1950s, liquid scintillation counting $(\mathrm{LSC})^{1)}$ has been a popular and indispensable technique in the detection and quantitative measurement of $\beta$ emitters needed in environmental and life sciences studies. The count rate of samples depends on how the energy from nuclear decay events is efficiently converted to detectable light flashes by a liquid scintillation cocktail. Various chemical substances in the sample prevent energy being transferred to the chemically fluorescing molecules; other substances absorb fluorescence photons within the scintillator. This phenomenon is called "quenching" and is chemically based preventing energy transfer from nuclear decay to the fluorescing molecules. Chemical quenching can be corrected using a set of quenched standards, by which means the activity of a sample can be determined. Sets of ${ }^{3} \mathrm{H}$ and
${ }^{14} \mathrm{C}$ quenched standards are commercially available, whereas those of other radionuclides have to be prepared by researchers in each instance. As is known, the preparation of a set of quenched standards requires a specially designed laboratory to handle the various radioactive samples. Hence quenching correction methods without needing sets of quenched standards are sought to improve LSC usability. Researchers have reported various quenching correction methods (e.g., internal standard method $^{2-4)}$, conventional integral counting method (CICM) ${ }^{5,6}$, modified integral counting method $(\mathrm{MICM})^{7,8)}$, CIEMAT/ NIST $^{9,10)}$, efficiency tracing method ${ }^{11)}$ ).

The MICM was proposed by Homma et al. ${ }^{7)}$ who modified the CICM by extrapolating integral counting curves to the zero detection threshold (ZDT) rather than the zero pulse height. At the ZDT, the integral counts coincide with the radioactivity 
of the sample. They determined the ZDT by measuring an unquenched ${ }^{3} \mathrm{H}$ standard sample. Samples having the same amount of radioactive material give the same integral counts at the ZDT. If various quenched samples having the same amount of radioactive material can be prepared, the disintegration rate can be determined by a procedure like the MICM. In this method, the integral counts of various quenched samples should converge to a point because of the regularity of quenching, and the point of convergence indicates the disintegration rate of the samples. However, severely quenched samples prevent integral counts of samples from converging ${ }^{12)}$.

We evaluated the MICM with various quenched samples (MICM-VQ) and various scintillators to assess the applicability of the MICM-VQ for other scintillators.

\section{Experimental}

\section{2-1. MICM for various quenched samples}

The prepared liquid scintillation cocktails each contained a radioactive sample of the same amount but a quencher of differing amounts. The scintillation spectra of these prepared cocktails were measured using a liquid scintillation counter (LS counter) and were converted to integral scintillation spectra using the formula for the integral count rate $I C$ (chi) at a given integral channel number chi

$$
I C(c h i)=\sum_{c h=c h i}^{\beta \max } C(c h)
$$

where ch is the channel number, which is proportional to the $\beta$ particle energy, and $C(c h)$ is the count rate at the given channel. The integral scintillation spectra are plots of the integral count rate against the integral channel number, respectively. In MICM, the observed integral counts of a ${ }^{3} \mathrm{H}$ unquenched standard are plotted at several integral channel numbers and the curve is then extrapolated to the integral count rate, which is equivalent to its disintegration rate. The integral channel number at this integral count rate represents the ZDT. In MICM-VQ, quadratic regression curves, which are obtained by the least- squares method, are drawn on the integral scintillation spectra curves ${ }^{12)}$. Where the quadratic regression curve fits the integral scintillation spectrum curve corresponds to the area above 50 chi. This area is selected because the low-channel area below the peak of the scintillation spectrum is affected by poor detection of low energy $\beta$ particle by LS counter. In previous paper ${ }^{12)}$ the high channel number was initially set to be 1000 chi and the fitted function was examined by the adjusted $R^{2} \geq 0.9997$, that is, in $R^{2}<0.9997$ from 50 chi to 1000 chi, the high channel number was reduced to be satisfied $R^{2} \geq 0.9997$. In this paper, however, the high channel number was found to satisfy $R^{2} \geq 0.9997$, not set the upper limit of 1000 chi because the disintegration rate of unknown sample was calculated more precisely. The curves of the quadratic regression converge to a point, which is determined from the minimum relative standard deviation $(\leq 0.2 \%)$ of the fitting functions for a given integral channel number. The integral count rate at the point of convergence indicates the actual disintegration rate for the samples.

\section{2-2. Reagents of radionuclides}

\section{2-2-1. Carbon-14}

For ${ }^{14} \mathrm{C}$, three sets of quenched standards were used to understand the influence of the scintillators. Details are listed in Table 1. The samples were sealed in glass vials. Spectral data for Ultima Gold ${ }^{\mathrm{TM}}$ obtained from a previous report were used ${ }^{12)}$.

\section{2-2-2. Sulfur-35}

For ${ }^{35} \mathrm{~S}$, sodium sulfate aqueous solution labeled by ${ }^{35} \mathrm{~S}$ as source was purchased from Japan Radioisotope Association (PerkinElmer). The radioactivity of the solution was diluted to $7 \mathrm{kBq} \mathrm{mL} L^{-1}$. One milliliter of solution was inserted into a 20-mL plastic vial (PerkinElmer, Super Polyethylene Vial with Quick Closure), and the six sample vials were ready in total. To each sample vial, $0,1,2,3,4,5 \mathrm{~mL}$ of ultrapure water was added as a quencher. Either Ecoscint ${ }^{\mathrm{TM}} \mathrm{XR}$ or Ultima Gold ${ }^{\mathrm{TM}}$ $\mathrm{AB}$ scintillator was added to bring the total sample volume to $15 \mathrm{~mL}$. To calculate uncertainties for this procedure, the masses

Table 1 Specifications of the sets of ${ }^{14} \mathrm{C}$ quenched standards

\begin{tabular}{l|ccc}
\hline Manufacturer & Base solvent & Disintegration rate $(\mathrm{kdpm})$ & Relative uncertainty $(\%)$ \\
\hline ALOKA & Toluene & 77.3 & - \\
PACKARD* & Toluene & 128.7 & 1.0 \\
PerkinElmer & Di-isopropylnaphthalene & 122.2 & 1.0 \\
\hline
\end{tabular}

* PerkinElmer, Inc. acquired Packard Bioscience in April 2002. 
Table 2 Constituents of liquid scintillators

\begin{tabular}{|c|c|c|c|}
\hline \multirow{2}{*}{$\begin{array}{l}\text { Liquid } \\
\text { scintillator }\end{array}$} & \multicolumn{3}{|c|}{ Constituents* } \\
\hline & Solvents and auxiliary agents & First solute & Second solute \\
\hline \multirow{4}{*}{$\begin{array}{c}\text { Ecoscint }^{T M} \\
X R\end{array}$} & Mixture of Phenylethylxylenes & \multirow[b]{4}{*}{ (PPO) } & \multirow[b]{4}{*}{ (POPOP) } \\
\hline & Primary alcohol ethoxylates & & \\
\hline & 2-Butoxyethanol & & \\
\hline & Nonyl phenol poly (ethylene oxy) ethanol & & \\
\hline \multirow{3}{*}{$\begin{array}{c}\text { Ultima } \\
\text { Gold'M AB }\end{array}$} & Di-isopropylnaphthalene & \multirow{3}{*}{ PPO } & \\
\hline & Ethoxylated alkyl phenol & & \\
\hline & Butyl carbitol & & (bis-MSB) \\
\hline
\end{tabular}

* Data for each reagent was extracted from the Safety Data Sheet (SDS).

of solution, water, and scintillator composing the sample were measured ${ }^{12)}$. The constituents of the scintillators are listed in Table 2. The solvents that are relevant to chemical quenching are different whereas the solutes relevant to color quenching are almost the same. To understand the scintillator dependence in the MICM-VQ, the spectral data of Ultima Gold ${ }^{\mathrm{TM}} \mathrm{AB}$ were used $^{12)}$.

\section{2-2-3. Instruments}

The scintillation spectra were measured using a LS counter (ALOKA LSC-5100), which has a 4096-channel pulse-height analyzer. The external standard channels ratio (ESCR) was adopted as a quench-indicating parameter. The ESCR decreases with increasing quenching.

\section{Results and Discussions}

\section{3-1. Integral scintillation spectra from the sets of ${ }^{14} \mathrm{C}$} quenched standards

For the integral scintillation spectra of ${ }^{14} \mathrm{C}$ (left column in Fig. 1), the integral count rates are shown as light gray bold lines and the quadratic regression curves are drawn as colored solid lines. In Fig. 1 (c), the Ultima Gold ${ }^{\mathrm{TM}}$ spectral data were used $^{12)}$; however, the shape of the quadratic regression curves is slightly different from the corresponding figure in that report because there the high channel number used in quadratic regression fitting was set equal to or less than 1000 chi. The slope of the quadratic regression curve steepens with increasing quenching. To determine the point of convergence, the integral scintillation spectra were enlarged (right column in Fig. 1). Because severely quenched samples prevent convergence to a point, some quadratic regression curves associated with low ESCR have been omitted from the evaluation of the point of convergence. The main reason is that the shape of the scintillation spectra from the set of ${ }^{14} \mathrm{C}$ quenched standards manufactured by PACKARD (Fig. 2) in the low pulse-height region is constrained by a discrimination level and a coincidence unit in the LS counter. Therefore integral spectra of severely quenched samples are significantly affected by components of the LS counter in comparison with that of less quenched samples. In the MICM-VQ, the influence from components of a LS counter prevents a point of convergence occurring. In regard to quenching correction curves, the appropriate quadratic curves were chosen from three sets of quenched standards (Fig. 3). The three quenching correction curves are very similar and from each curve, the part of the quadratic regression curve for which the counting efficiency was more than about $90 \%$, was selected as input for the MICM-VQ to determine the point of convergence. That is, for the ALOKA samples, the PACKARD samples, and the PerkinElmer samples, the points of convergence were obtained using the quadratic regression curves for which the ESCR was equal to or higher than 2.05, 2.06, and 2.06 , respectively. The disintegration rates obtained and these relative uncertainties are $79.7 \mathrm{kdpm} \pm 1.4 \%$ for the ALOKA sample, $129.9 \mathrm{kdpm} \pm 1.3 \%$ for the PACKARD sample, and $123.8 \mathrm{kdpm} \pm 1.3 \%$ for the PerkinElmer sample in the coverage factor $k=2$. These values almost agree with assayed values (Table 1). This demonstrates that the MICM-VQ is adaptable to various scintillators and yields accurate disintegration rates when quenching in samples is not severe. 

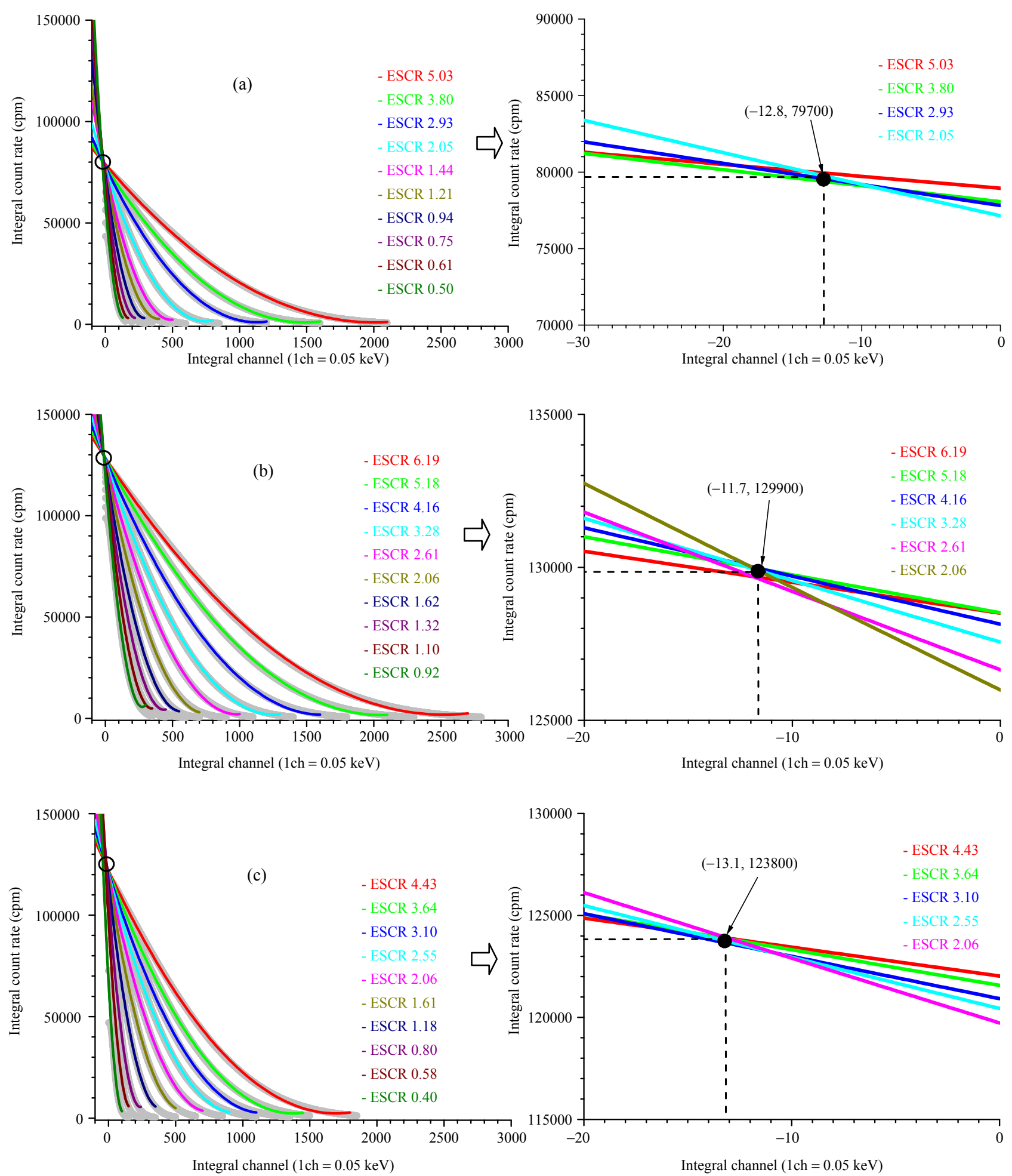

Fig. 1. Integral scintillation spectra of ${ }^{14} \mathrm{C}$ (left column) and an enlargement around the point of convergence (right column) for (a) ALOKA, (b) PACKARD, and (c) PerkinElmer. The light gray bold lines and colored solid lines represent the integral counts and the quadratic regression curves, respectively. 


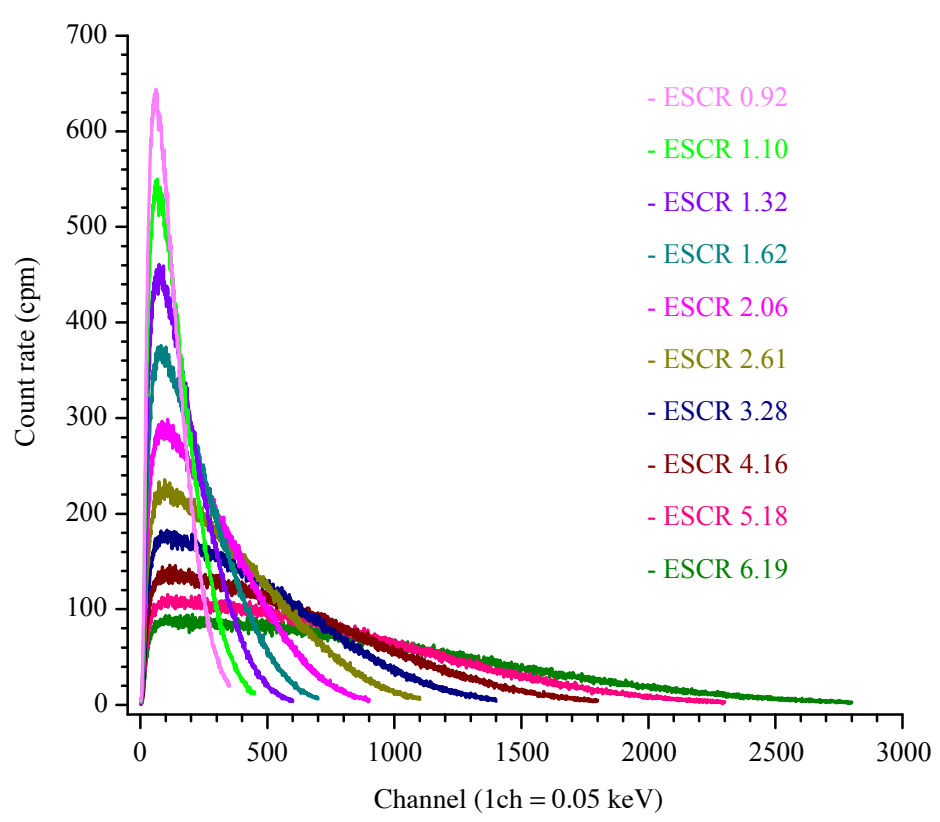

Fig. 2. Scintillation spectra of a set of ${ }^{14} \mathrm{C}$ quenched standards manufactured by PACKARD.

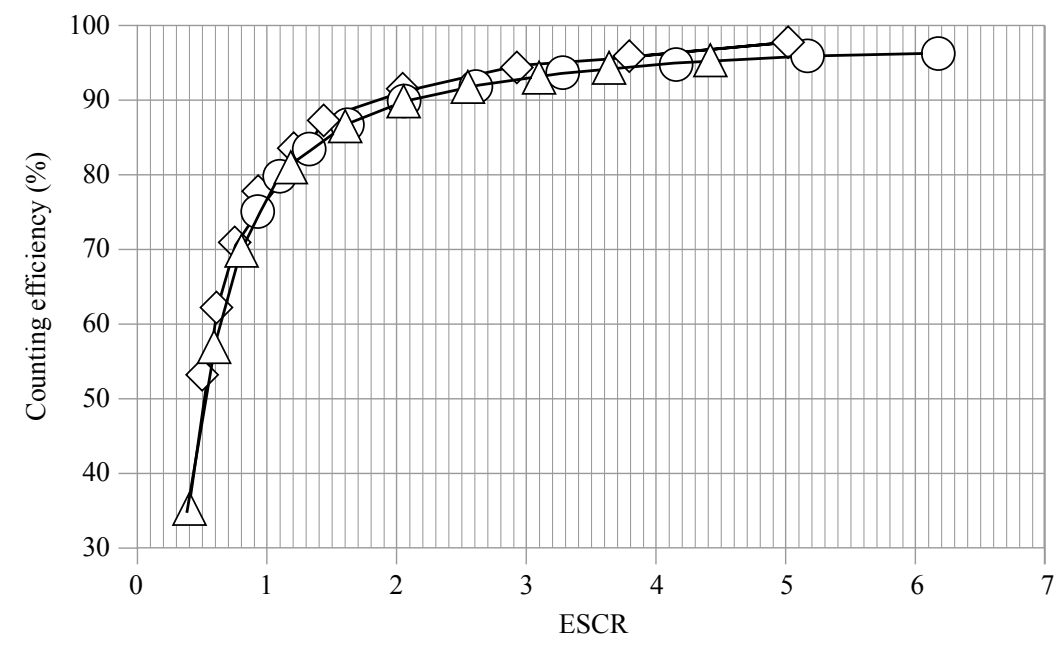

Fig. 3. Quenching correction curves for sets of ${ }^{14} \mathrm{C}$ quenched standards. $\diamond$ ALOKA $\bigcirc$ PACKARD $\triangle$ PerkinElmer

3-2. The integral scintillation spectra from ${ }^{35} \mathrm{~S}$ quenched sample sets

The integral scintillation spectra obtained for ${ }^{35} \mathrm{~S}$ are given in the left column in Fig. 4. In Fig. 4(b), Ultima Gold ${ }^{\mathrm{TM}} \mathrm{AB}$ spectral data were used ${ }^{12)}$, although again the shape of the quadratic regression curves is slightly different for the same reason as previously mentioned in Section 3.1. In regard to determining the point of convergence, the integral scintillation spectra were enlarged (right column in Fig. 4). For Ecoscint ${ }^{\mathrm{TM}}$ $\mathrm{XR}$ and Ultima Gold ${ }^{\mathrm{TM}} \mathrm{AB}$ samples, convergence was obtained from the quadratic regression curves for which the ESCR was equal to or higher than 2.30 and 2.92, respectively, and the ESCRs were slightly higher compared with those in ${ }^{14} \mathrm{C}$ determination. Values of the disintegration rates and relative 

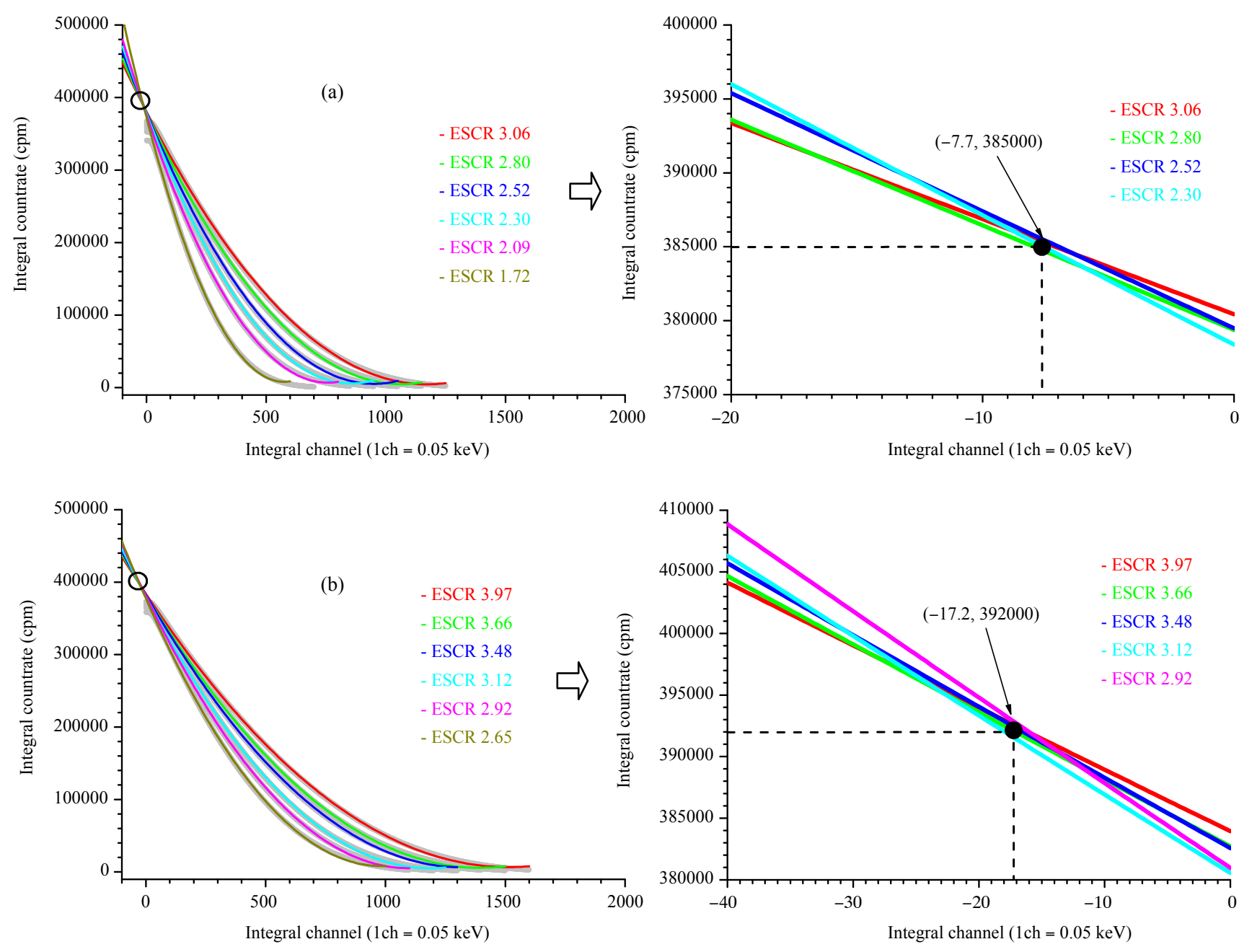

Fig. 4. Integral scintillation spectra of ${ }^{35} \mathrm{~S}$ and enlargement around the point of convergence for (a) Ecoscint ${ }^{\mathrm{TM}} \mathrm{XR}$ and (b) Ultima Gold ${ }^{\mathrm{TM}}$ AB. The light gray bold lines and the colored solid lines represent the integral counts and the quadratic regression curves, respectively.

Table 3 Measurement values of disintegration rates and these relative uncertainties for ${ }^{35} \mathrm{~S}$ samples

\begin{tabular}{|c|c|c|c|c|}
\hline \multirow{2}{*}{$\begin{array}{l}\text { Scintillation } \\
\text { cocktail }\end{array}$} & \multicolumn{2}{|c|}{ MICM-VQ } & \multicolumn{2}{|c|}{${ }^{14} \mathrm{C}$ quenching correction curve } \\
\hline & $\begin{array}{l}\text { Measurement value of } \\
\text { disintegration rate }(\mathrm{kdpm})\end{array}$ & $\begin{array}{c}\text { Relative } \\
\text { uncertainty* }(\%)\end{array}$ & $\begin{array}{l}\text { disintegration } \\
\text { rate }(\mathrm{kdpm})\end{array}$ & $\begin{array}{c}\text { Relative } \\
\text { uncertainty* (\%) }\end{array}$ \\
\hline$E_{\cos c i n t}{ }^{T M} X R$ & 385 & 0.7 & $\begin{array}{l}383 \text { (ALOKA) } \\
391 \text { (PACKARD) }\end{array}$ & $\begin{array}{l}1.6(\text { ALOKA) } \\
1.1(\text { PACKARD) }\end{array}$ \\
\hline Ultima Gold ${ }^{T M} A B$ & 392 & 0.8 & 393 (PerkinEImer) & 2.0 (PerkinEImer) \\
\hline
\end{tabular}

* The coverage factor of relative uncertainties is two $(k=2)$

uncertainties of the samples are listed in Table 3. Two values obtained agree well. The disintegration rates of the samples also were determined from ESCR data. Buckley ${ }^{13)}$ reported that the quenching correction curve of ${ }^{14} \mathrm{C}$ can be applied in the ${ }^{35} \mathrm{~S}$ analysis as a secondary counting standard. The ${ }^{14} \mathrm{C}$ curves in Fig. 3 were used. The disintegration rates of Ecoscint ${ }^{\mathrm{TM}} \mathrm{XR}$ and Ultima Gold $^{\mathrm{TM}} \mathrm{AB}$ samples were calculated using ALOKA or PACKARD curve and PerkinElmer curve, respectively, because 
the scintillator constituents of each sample were similar to those of each ${ }^{14} \mathrm{C}$ quenched standard set. The values obtained using the MICM-VQ also agreed well with those using ESCR. In contrast, the activity of ${ }^{35} \mathrm{~S}$ sample using Ultima Gold ${ }^{\mathrm{TM}} \mathrm{AB}$ was determined using the CIEMAT/NIST method ${ }^{12)}$. The activity of ${ }^{35} \mathrm{~S}$ and its relative uncertainty is found to be $402 \mathrm{kdpm} \pm 1.4 \%$ in the coverage factor $k=2$. The results indicate that the MICM-VQ has great potential in the determination of the disintegration rate of $\beta$ emitters without needing a standard sample of a particular nuclide and can also be adopted for various scintillators.

\section{Conclusions}

The applicability of the MICM-VQ for the determination of the disintegration rate of $\beta$ emitters $\left({ }^{14} \mathrm{C}\right.$ and $\left.{ }^{35} \mathrm{~S}\right)$ was investigated using several different types of scintillator. The ESCR parameter, which is exploited for this determination differs in value according to radioisotopes and scintillators. If the samples are not quenched severely, MICM-VQ is useful for the determination of the disintegration rates of unknown samples, is not influenced by scintillator type, and requires no standard samples.

\section{Acknowledgments}

The authors thank Masato Mae and Chika Katayama, graduates of University of Toyama for their participation in this study as part fulfillment of graduate work.

\section{References}

1) L'Annunziata, M. F.: Chapter 5 Liquid Scintillation Analysis: Principles and Practice, Handbook of Radioactivity Analysis Second Edition, Academic Press, USA, pp. 347-535 (2003).

2) Hendee, W. R., Ibbott, G. S., Crusha, K. L.: ${ }^{3} \mathrm{H}$-toluene, ${ }^{3} \mathrm{H}$-water and ${ }^{3} \mathrm{H}$-hexadecane as internal standards for toluene- and dioxanebased liquid scintillation cocktail, Int. J. Appl. Radiat. Isot., 23, 90-95 (1972).

3) Dobbs, H. E.: Dispersing Solutions for Liquid Scintillation Counting, Memorandum NO. AERE-M1574, UK Atomic Energy Authority, Harwell (1965).

4) Thomas, R. C., Judy, R. W., Harpootlian, H.: Dispenser for addition of internal standard in liquid scintillation counting, Anal. Biochem., 13, 358-360 (1965).

5) Goldstein, G.: Absolute liquid-scintillation counting of beta emitters, Nucleonics, 23, 67-69 (1965).

6) Homma, Y., Murakami, Y.: Study on the applicability of the integral counting method for the determination of ${ }^{226} \mathrm{Ra}$ in various sample forms using a liquid scintillation counter, J. Radioanal. Chem., 36, 173-184 (1977).

7) Homma, Y., Murase, Y., Handa, K.: The zero detection threshold of a liquid scintillation spectrometer and its application to liquid scintillation counting, Appl. Radiat. Isot., 45, 341-344 (1994).

8) Homma, Y., Murase, Y., Handa, K.: Absolute liquid scintillation counting of ${ }^{35} \mathrm{~S}$ and ${ }^{45} \mathrm{Ca}$ using a modified integral counting method, J. Radioanal. Nucl. Chem. Letters, 187, 367-374 (1994).

9) Günther, E.: Standardization of the EC nuclides ${ }^{55} \mathrm{Fe}$ and ${ }^{65} \mathrm{Zn}$ with the CIEMAT/NIST LSC tracer method, Appl. Radiat. Isot., 49 (9-11), 1055-1060 (1998).

10) Sato, Y., Ishizu, H. and Yamada, T.: Standardization of ${ }^{14} \mathrm{C}$ by CIEMAT-NIST Method and TDCR Method, Radioisotopes, 65, $1-5$ (2016).

11) Takiue, M. and Ishikawa, H.: Thermal neutron reaction cross section measurements for fourteen nuclides with a liquid scintillation spectrometer, Nucl. Instrum. Methods, 148, 7 157161 (1978)

12) Hara, M., Nakayama, M., Hirokami, K., Aso, T.: Appropriate quenching level in modified integral counting method by liquid scintillation counting, J. Radioanal. Nucl. Chem., 310, 857-863 (2016).

13) Buckley, J. P.: The use of Carbon-14 as a secondary counting standard for sulphur-35, Int. J. Appl. Radiat. Isot., 22, 41-42 (1971). 